\title{
A Coupled Approach to Solve the Family of Kuramoto-Sivashinsky Equations
}

\author{
PRATIBHA JOSHI ${ }^{1}$ AND MAHESHWAR PATHAK ${ }^{2}$ \\ ${ }^{1,2}$ Department of Mathematics \\ School of Engineering \\ University of Petroleum and Energy Studies (UPES) \\ Energy Acres, VPO Bidholi, PO Prem Nagar, Dehradun 248007, Uttarakhand \\ INDIA
}

\begin{abstract}
This paper presents a coupled approach to solve the Kuramoto-Shivashinsky equations. This approach is a combination of modified variation iteration method and a rational approximation by mathematical software MATHEMATICA. Numerical examples illustrate that this combination of two techniques improves accuracy.
\end{abstract}

Keywords: Kuramoto-Shivashinsky equations; variational iteration method (VIM); modified variational iteration method (MVIM); rational approximation

Received: February 16, 2020. Revised: July 30, 2020. Accepted: August 7, 2020. Published: August 10, 2020.

\section{Introduction}

Nonlinear equations arise in many fields e.g. chemical engineering, fluid dynamics, mechanics, nuclear physics, biology etc. It has always been challenging to find an accurate solution of nonlinear problems because of their complexity and varieties. The main problem with mathematical methods in solving nonlinear equations is that one method can work in one type of equation and may not work in another. Hence many researchers and mathematicians are using coupled approaches to solve them to get the maximum benefit.

Kuramoto-Shivashinsky equation (KSequation) is a very important nonlinear partial differential equation with wide applications in engineering and science. It models chaotic behavior arising in various fields such as thin film flow, phase turbulence in chemical oscillations, plasma physics, two phase flows etc. Due to its vast applications, a great number of studies are going on in finding highly accurate solution of Kuramoto-Shivashinsky equation.

In [1], Porshokouhi et al. applied original variational iteration method[13] to solve KuramotoShivashinsky equation. Variational iteration method $[13-16,21]$ is a very powerful iterative method for the solution of nonlinear ordinary and partial differential equations. The disadvantage of VIM method is after few iterations, it becomes very difficult to proceed further even by mathematical softwares because of repeated calculations which increase computational time. Hence, many modified variational iteration methods (MVIM) $[2-5,18-20$, 22] have been introduced which take shorter time and give same or better accuracy. One of the modified version of VIM is given by Abassy et al. [2]. By this modified version, approximated power series solutions are obtained that converge in a neighborhood of the initial point. It reduces the repeated calculations and so the computational time. But, a major drawback of this method is a very short region of convergence. Hence, in [3], Abassy etal. coupled MVIM with pade technique to enlarge the region of convergence.

In this paper, MVIM and rational approximation by MATHEMATICA have been coupled. It can be an alternate way to enlarge the region of convergence of MVIM and it gives better accuracy at some points also.

\section{Kuramoto-Shivashinsky equation}

In the present paper, the following Generalized Kuramoto- Sivashinsky (GKS) equation has been considered

$\frac{\partial \psi}{\partial t}+\psi \frac{\partial \psi}{\partial \zeta}+a \frac{\partial^{2} \psi}{\partial \zeta^{2}}+b \frac{\partial^{3} \psi}{\partial \zeta^{3}}+c \frac{\partial^{4} \psi}{\partial \zeta^{4}}=0$

with initial condition $\psi(\zeta, 0)=r(x)$.

Here $a, b$ and $c$ are constants. For $b=0$, equation (1) represents the Kuramoto-Sivashinsky 
(KS) equation. In [17], the function $\psi(\zeta, t)$ describes the position of the front at time $\boldsymbol{t}$, at height $\psi$ above the point $\zeta$ at the bottom of the cylinder for flame front in a cylinder.

Kuramoto-Sivashinsky (KS) equation is widely used to describe so many physical phenomena[6-12, 23].

\section{Modified Variational Iteration Method}

In this section, a brief description of modified variational iteration method[2] has been given. Let us consider a nonlinear problem

$$
\begin{aligned}
& \operatorname{L\psi }(\zeta, t)+R \psi(\zeta, t)+N \psi(\zeta, t)=p(\zeta, t) \\
& \psi(\zeta, 0)=r_{0}(\zeta) \\
& \left.\frac{\partial \psi(\zeta, t)}{\partial t}\right|_{t=0}=r_{1}(x) \\
& \cdot \\
& \cdot \\
& \left.\frac{\partial^{\mu-1} \psi(\zeta, t)}{\partial t^{\mu-1}}\right|_{t=0}=r_{\mu-1}(x)
\end{aligned}
$$

where $L=\frac{\partial^{\mu}}{\partial t^{\mu}}, \mu=1,2,3 \ldots$ is the highest partial derivative with respect to $\mathrm{t}, R$ is a linear operator and $N \psi(\zeta, t)$ is the nonlinear term. $R \psi(\zeta, t)$ and $N \psi(\zeta, t)$ are free of partial derivatives with respect to $t$ and $p(\zeta, t)$ is the non-homogeneous term.

In the variational iteration method [13] we use the following iteration formula:

$\Psi_{v+1}(x, t)=\Psi_{v}(x, t)+\int_{0}^{t} \lambda\left\{L \Psi_{v}+\overline{R \Psi_{v}}+\overline{N \Psi_{v}}-p\right\} d \eta$

where $\lambda$ is called a general Lagrange multiplier [24] which is determined optimally via variational theory, $\overline{R \Psi_{v}}$ and $\overline{N \Psi_{v}}$ are considered as restricted variations i.e. $\delta \overline{R \Psi_{v}}=0, \delta \overline{N \Psi_{v}}=0$.

On calculating variation with respect to $\Psi_{v}$, we get

$$
\delta \Psi_{v+1}(\zeta, t)=\delta \Psi_{v}(\zeta, t)+\delta \int_{0}^{t} \lambda(\eta)\left\{L \Psi_{v}+\overline{R \Psi_{v}}+\overline{N \Psi_{v}}-p\right\} d \eta
$$

$=\delta \Psi_{v}(\zeta, t)+\delta \int_{0}^{t} \lambda(\eta) L \Psi_{v} d \eta$

$=\delta \Psi_{v}(\zeta, t)+\delta \int_{0}^{t} \lambda(\eta) \frac{\partial^{\mu} \Psi_{v}}{\partial \eta^{\mu}} d \eta$

$=\delta \Psi_{v}(\zeta, t)+\left.\delta \frac{\partial^{\mu-1} \Psi_{v}(\zeta, \eta)}{\partial \eta^{\mu-1}}(\lambda(\eta))\right|_{\eta=t}-\left.\delta \frac{\partial^{\mu-2} \Psi_{v}(\zeta, \eta)}{\partial \eta^{\mu-2}}\left(\lambda^{\prime}(\eta)\right)\right|_{\eta=t}$

$+\left.\delta \frac{\partial^{\mu-3} \Psi_{v}(\zeta, \eta)}{\partial \eta^{s-3}}\left(\lambda^{\prime \prime}(\eta)\right)\right|_{\eta=t}-\cdots+\left.(-1)^{\mu-1} \delta \Psi_{v}(\zeta, \eta) \frac{\partial^{\mu-1} \lambda(\eta)}{\partial \tau^{\mu-1}}\right|_{\eta=t}$

$+(-1)^{\mu} \int_{0}^{t} \delta \Psi_{v}(\zeta, \eta) \frac{\partial^{\mu} \lambda(\eta)}{\partial \tau^{\mu}} d \eta$

$\delta \Psi_{v+1}(\zeta, t)=\left.\delta \Psi_{v}(\zeta, \eta)\left(1+(-1)^{\mu-1} \frac{\partial^{\mu-1} \lambda(\eta)}{\partial \eta^{\mu-1}}\right)\right|_{\eta=t}+\left.\delta \frac{\partial^{\mu-1} \Psi_{v}(\zeta, \eta)}{\partial \eta^{\mu-1}}(\lambda(\eta))\right|_{\eta=t}$

$-\left.\delta \frac{\partial^{\mu-2} \Psi_{v}(\zeta, \eta)}{\partial \eta^{\mu-2}}\left(\lambda^{\prime}(\eta)\right)\right|_{\eta=t}+\left.\delta \frac{\partial^{\mu-3} \Psi_{v}(\zeta, \eta)}{\partial \tau^{\mu-3}}\left(\lambda^{\prime \prime}(\eta)\right)\right|_{\eta=t}$

$-\cdots+(-1)^{\mu} \int_{0}^{t} \delta \Psi_{v}(\zeta, \eta) \frac{\partial^{\mu} \lambda(\eta)}{\partial \eta^{\mu}} d \eta$

From equation (4) following stationary conditions are obtained:

$\delta \psi_{v}: \lambda^{(\mu)}(\eta)=0$,

$\delta \psi_{v}^{(\mu-1)}:\left.\lambda(\eta)\right|_{\eta=t}=0$,

$\delta \psi_{v}^{(\mu-2)}:\left.\lambda^{\prime}(\eta)\right|_{\eta=t}=0$,

$\delta \psi_{v}: 1+\left.(-1)^{(\mu-1)} \lambda^{(\mu-1)}(\eta)\right|_{\eta=t}=0$.

Therefore, the Lagrange multiplier is obtained as:

$$
\lambda=-\frac{(t-\eta)^{(\mu-1)}}{(\mu-1) !}
$$

Now substituting the value of $\lambda$ from equation (5) to (2), we get

$\Psi_{v+1}(\zeta, t)=\Psi_{v}(\zeta, t)+\int_{0}^{t}-\frac{(t-\eta)^{(\mu-1)}}{(\mu-1) !} \cdot\left\{L \Psi_{v}+R \Psi_{v}+N \Psi_{v}-p\right\} d \eta$

Equation (6) becomes the formulation of variational iteration method(VIM). Equation (6) can be solved iteratively using initial condition $\Psi_{0}(\zeta, t)=r_{0}(\zeta)+r_{1}(\zeta) t+\cdots+r_{\mu-1}(\zeta) t^{\mu-1}$. 
To stop repeated calculations by formula (6), the following modification in formula (6) is suggested [2]:

$\Psi_{v+1}(\zeta, t)=\Psi_{0}(\zeta, t)+\int_{0}^{t} \lambda(\eta)\left\{R \Psi_{v}(\zeta, \eta)+N \Psi_{v}(\zeta, \eta)-p(\zeta, \eta)\right\} d \eta$

where $\lambda$ is given by equation (5).

By this modification some repeated calculations are removed. To deal with the nonsettled terms[2] which slow the convergence down following modification is suggested [2] in formula (7):

$\Psi_{v+1}(\zeta, t)=\Psi_{0}(\zeta, t)+\int_{0}^{t} \lambda(\eta)\left\{R \Psi_{v}(\zeta, \eta)+\Omega_{v}(\zeta, \eta)-p(\zeta, \eta)\right\} d \eta$

where $\Psi_{0}(\zeta, t)=r_{0}(\zeta)+r_{1}(\zeta) t+\cdots+r_{\mu-1}(\zeta) t^{\mu-1}$ and $\Omega_{v}(\zeta, \eta)$ are obtained from

$N \Psi_{v}(\zeta, t)=\Omega_{v}(\zeta, t)+O\left(t^{v+1}\right)$.

To stop all the repeated calculations, formula (8) is rewritten as [2]:

$$
\begin{aligned}
& \Psi_{v+1}(\zeta, t)=\Psi_{0}(\zeta, t)+\int_{0}^{t} \lambda(\eta)\left\{R \Psi_{v-1}(\zeta, \eta)\right. \\
& \left.+\Omega_{v-1}(\zeta, \eta)-p(\zeta, \eta)\right\} d \eta+\int_{0}^{t} \lambda(\eta)\left\{R \left(\Psi_{v}(\zeta, \eta)\right.\right. \\
& \left.\left.-\Psi_{v-1}(\zeta, \eta)\right)+\left(\Omega_{v}(\zeta, \eta)-\Omega_{v-1}(\zeta, \eta)\right)\right\} d \eta
\end{aligned}
$$

From equation (8), it can be written as:

$$
\begin{aligned}
& \Psi_{v}(\zeta, t)=\Psi_{0}(\zeta, t)+\int_{0}^{t} \lambda(\eta)\left\{R \Psi_{v-1}(\zeta, \eta)\right. \\
& \left.+\Omega_{v-1}(\zeta, \eta)-p(\zeta, \eta)\right\} d \eta
\end{aligned}
$$

From equations (10) and (11), it can be written as:

$$
\begin{aligned}
& \Psi_{v+1}(\zeta, t)=\Psi_{v}(\zeta, t)+\int_{0}^{t} \lambda(\eta)\left\{R\left(\Psi_{v}(\zeta, \eta)-\Psi_{v-1}(\zeta, \eta)\right)\right. \\
& +\left(\Omega_{v}(\zeta, \eta)-\Omega_{v-1}(\zeta, \eta)\right) d \eta
\end{aligned}
$$

where

$$
\begin{aligned}
& \Psi_{-1}=0, \Psi_{0}=r_{0}(\zeta)+r_{1}(\zeta) t+\cdots+r_{\mu-1}(\zeta) t^{\mu-1}, \\
& \Psi_{1}=\Psi_{0}-\int_{0}^{t}\left\{R\left(\Psi_{0}-\Psi_{-1}\right)+\left(\Omega_{0}-\Omega_{-1}\right)-p\right\} d \eta,
\end{aligned}
$$

and $\Omega_{v}(\zeta, t)$ is obtained from

$$
N \Psi_{v}(\zeta, t)=\Omega_{v}(\zeta, t)+O\left(t^{v+1}\right) .
$$

Equation (12) is called modified variational iteration method [2]. In this formula no repeated calculation is used.

\section{Coupling of modified variational iteration method and rational approximations by MATHEMATICA}

In this paper, we have coupled MVIM with rational approximation by mathematical software MATHEMATICA to solve Kuramoto-Sivashinsky equation. Firstly, MVIM [2] has been applied to the problem and then to enlarge the region of convergence rational approximation by "Function Approximation Package" of MATHEMATICA has been used. Although there are many options in MATHEMATICA for finding rational approximation but it has been seen that in this case the approximations by the commands "RationalInterpolation" and

“EconomizedRationalApproximation" give the best results.

The command "RationalInterploation" in MATHEMATICA given in the form

RationalInterpolation[expr, $\left.\{\zeta, \kappa, v\},\left\{\zeta_{1}, \zeta_{2}, \cdots, \zeta_{\kappa+v+1}\right\}\right]$ returns the rational interpolant to "expr" with numerator order $\kappa$ and denominator order $v$, where $\zeta_{1}, \zeta_{2}, \cdots$ are the abscissas of the interpolation points. The command "EconomizedRationalApproximation"given in the form

EconomizedRationalApproximation[expr, $\left.\left\{\zeta,\left\{\zeta_{0}, \zeta_{1}\right\}, \kappa, v\right\}\right]$ gives the economized rational approximation to "expr" that is good over the interval $\zeta_{0}$ to $\zeta_{1}$ with numerator order $\kappa$ and denominator order $v$.

In our coupled approach, after every iteration in MVIM, we find the rational approximation of the solution of the problem using these two commands of MATHEMATICA discussed above.

\section{Numerical Examples}

In this section, three numerical examples of Kuramoto-Sivashinsky equation have been solved using coupling of MVIM and rational approximation technique by MATHEMATICA. To show the efficiency of this approach comparison has been made with original VIM, modified VIM, and coupling of modified VIM and Pade approximation.

Example 1: Consider the Kuramoto-Sivashinsky equation: 


$$
\frac{\partial \psi}{\partial t}+\psi \frac{\partial \psi}{\partial \zeta}+\frac{\partial^{2} \psi}{\partial \zeta^{2}}+\frac{\partial^{4} \psi}{\partial \zeta^{4}}=0
$$

with initial condition

$$
\begin{aligned}
& \psi(\zeta, 0)=\omega+\frac{5}{19} \sqrt{\frac{11}{19}}\left[11 \tanh ^{3}\left(q\left(\zeta-\zeta_{0}\right)\right)\right. \\
& \left.-9 \tanh \left(q\left(\zeta-\zeta_{0}\right)\right)\right]
\end{aligned}
$$

\section{Solution by VIM :}

By applying original variational iteration method the following correctional functional [1] can be constructed:

$$
\begin{aligned}
& \psi_{v+1}(\zeta, t)=\psi_{v}(\zeta, t)+\int_{0}^{t} \lambda(\eta)\left\{\frac{\partial \psi_{v}(\zeta, \eta)}{\partial \eta}\right. \\
& \left.+\overline{\psi_{v}}(\zeta, \eta) \frac{\partial \overline{\psi_{v}}(\zeta, \eta)}{\partial \zeta}+\frac{\partial^{2} \overline{\psi_{v}}(\zeta, \eta)}{\partial \zeta^{2}}+\frac{\partial^{4} \overline{\psi_{v}}(\zeta, \eta)}{\partial \zeta^{4}}\right\} d \eta
\end{aligned}
$$

where $\lambda(\eta)$ is the Lagrange multiplier and is evaluated by applying variational theory and $\psi_{v}$ is $v^{\text {th }}$ approximate solution and $\overline{\psi_{v}}$ denotes a restricted variation[i.e. $\delta \overline{\psi_{v}}=0$ ].

Now applying the variation with respect to $\psi_{v}$ we get the following stationary conditions

$$
\begin{aligned}
& 1+\left.\lambda(\eta)\right|_{\eta=t}=0 \\
& \left.\lambda^{\prime}(\eta)\right|_{\eta=t}=0
\end{aligned}
$$

which gives $\lambda=-1$ and the correctional function becomes the following iteration formula:

$$
\begin{aligned}
& \psi_{v+1}(\zeta, t)=\psi_{v}(\zeta, t)-\int_{0}^{t}\left\{\frac{\partial \psi_{v}(\zeta, \eta)}{\partial \eta}\right. \\
& \left.+\psi_{v}(\zeta, \eta) \frac{\partial \psi_{v}(\zeta, \eta)}{\partial \zeta}+\frac{\partial^{2} \psi_{v}(\zeta, \eta)}{\partial \zeta^{2}}+\frac{\partial^{4} \psi_{v}(\zeta, \eta)}{\partial \zeta^{4}}\right\} d \eta
\end{aligned}
$$

Now using the initial approximation (14) and iteration formula (15) we can get the approximations of solution of equation (13).

From equation (12), the formulation of MVIM for the solution of equation (13) is

$$
\begin{aligned}
& \Psi_{v+1}(\zeta, t)=\Psi_{v}(\zeta, t)-\int_{0}^{t}\left\{\left(\frac{\partial^{2} \Psi_{v}(\zeta, \eta)}{\partial \zeta^{2}}-\frac{\partial^{2} \Psi_{v-1}(\zeta, \eta)}{\partial \zeta^{2}}\right)\right. \\
& \left.+\left(\frac{\partial^{4} \Psi_{v}(\zeta, \eta)}{\partial \zeta^{4}}-\frac{\partial^{4} \Psi_{v-1}(\zeta, \eta)}{\partial \zeta^{4}}\right)\right\} d \eta \\
& -\int_{0}^{t}\left(\Omega_{v}(\zeta, \eta)-\Omega_{v-1}(\zeta, \eta)\right) d \eta \\
& \text { where } \psi(\zeta, t) \cdot \frac{\partial \psi(\zeta, t)}{\partial \zeta}=\Omega_{v}(\zeta, t)+O\left(t^{n+1}\right) .
\end{aligned}
$$

Now to increase the region of convergence of MVIM, the rational interpolation and economized rational interpolation commands of the "FUNCTION APPROXIMATION PACKAGE" of MATHEMATICA on the series obtained by the formulation(16) have been applied. Also, to compare the results of our approach, we have also applied the coupled technique by Abbasy et.al.[3] to solve equation(13) in which after getting the series approximation at every iteration by formulation (16), its Pade approximation is evaluated. The error analysis for example-1 by using different techniques at arbitrary points has been shown in Table-1 for $\omega=0.1, q=\frac{1}{2} \sqrt{\frac{11}{19}}, \zeta_{0}=-10$ in first iteration.

Table -2 and Table 3 show error analysis for same data in second and third iteration respectively. The errors shown in the tables are absolute error evaluated using the exact solution of Example-1:

$$
\begin{aligned}
& \psi(\zeta, t)=\omega+\frac{5}{19} \sqrt{\frac{11}{19}}\left[11 \tanh ^{3}\left(q\left(\zeta-\omega t-\zeta_{0}\right)\right)\right. \\
& \left.-9 \tanh \left(q\left(\zeta-\omega t-\zeta_{0}\right)\right)\right]
\end{aligned}
$$

Table 1: Error Analysis of Example-1 in first iteration

\begin{tabular}{|l|l|l|l|}
\hline$(\mathrm{x}, \mathrm{t})$ & $(0.1,0.1)$ & $(0.2,0.2)$ & $(0.3,0.3)$ \\
\hline VIM & 0.000265499 & 0.000492857 & 0.00068613 \\
\hline MVIM & 0.000265499 & 0.000492857 & 0.00068613 \\
\hline MVIM+PADE & 0.000265499 & 0.000492856 & 0.000686127 \\
\hline MVIM+RAT & 0.00026531 & 0.000492316 & 0.000685588 \\
\hline MVIM+ECORAT & 0.000265304 & 0.000492316 & 0.000685592 \\
& & & \\
\hline
\end{tabular}


Table 2: Error Analysis of Example-1 in second iteration

\begin{tabular}{|l|l|l|l|}
\hline$(\mathrm{x}, \mathrm{t})$ & $(0.1,0.1)$ & $(0.2,0.2)$ & $(0.3,0.3)$ \\
\hline VIM & 0.000260423 & 0.000473721 & 0.000645612 \\
\hline MVIM & 0.000260421 & 0.000473711 & 0.000645585 \\
\hline MVIM+PADE & 0.000260421 & 0.000473711 & 0.000645583 \\
\hline MVIM+RAT & 0.000260232 & 0.000473167 & 0.000645038 \\
\hline MVIM+ECORAT & 0.000260226 & 0.000473168 & 0.000645043 \\
\hline
\end{tabular}

The Pade approximation, the rational interpolation and the economized rational interpolation by MATHEMATICA used in these calculations are evaluated of the same order $(2,2)$. The pade approximation is evaluated at $\zeta=0$ and the rational interpolation and the economized rational interpolation by MATHEMATICA are taken for the interval $(0,3)$.

Table 3: Error Analysis of Example-1 in third iteration

\begin{tabular}{|l|l|l|l|}
\hline$(\mathrm{x}, \mathrm{t})$ & $(0.1,0.1)$ & $(0.2,0.2)$ & $(0.3,0.3)$ \\
\hline VIM & 0.0002602760 & 0.00047276 & 0.000642981 \\
\hline MVIM & 0.0002602760 & 0.000472763 & 0.000642996 \\
\hline MVIM+PADE & 0.0002602760 & 0.000472763 & 0.000642993 \\
\hline MVIM+RAT & 0.000260086 & 0.00047222 & 0.000642449 \\
\hline MVIM+ECORAT & 0.00026008 & 0.00047221 & 0.00064245 \\
& & & \\
\hline
\end{tabular}

It can be seen from the tables 1, 2 and 3 that the results of the proposed schemes have good agreement with the results of VIM and MVIM and even they have slightly better results at these points. The comparison of results of tables 1,2 and 3 demonstrates that as we increase number of iterations the errors become lesser and the results of the proposed scheme become better than other methods.

Example 2: We have considered another form[1] of Kuramoto-Sivashinsky equation as second problem which is

$$
\frac{\partial \psi}{\partial t}+\psi \frac{\partial \psi}{\partial \zeta}-\frac{\partial^{2} \psi}{\partial \zeta^{2}}+\frac{\partial^{4} \psi}{\partial \zeta^{4}}=0
$$

with initial condition

$$
\begin{aligned}
& \psi(\zeta, 0)=\omega+\frac{15}{19 \sqrt{19}}\left[\tanh ^{3}\left(q\left(\zeta-\zeta_{0}\right)\right)\right. \\
& \left.-3 \tanh \left(q\left(\zeta-\zeta_{0}\right)\right)\right]
\end{aligned}
$$

Again, we apply all the techniques which we have applied for problem 1. In this problem we are considering the case of $\omega=0.2, q=\frac{1}{2 \sqrt{19}}, \zeta_{0}=-10$.

Table-4: Error analysis of Example-2 in first iteration

\begin{tabular}{|l|l|l|l|}
\hline$(\mathrm{x}, \mathrm{t})$ & $(0.3,0.3)$ & $(0.4,0.4)$ & $(0.5,0.5)$ \\
\hline VIM & 0.00140977 & 0.00181913 & 0.00220008 \\
\hline MVIM & 0.00140977 & 0.00181913 & 0.00220008 \\
\hline MVIM+PADE & 0.00140977 & 0.00181913 & 0.00220007 \\
\hline MVIM+RAT & 0.00140963 & 0.00181902 & 0.00220002 \\
\hline MVIM+ECORAT & 0.00140963 & 0.00181902 & 0.00220002 \\
& & & \\
\hline
\end{tabular}

The results of error analysis at some arbitrary points are displayed in Table-4. These errors are absolute errors. All rational approximants are taken of the same order and in the same interval as in case of Example-1. The exact solution of this problem is

$$
\begin{aligned}
& \psi(\zeta, t)=\omega+\frac{15}{19 \sqrt{19}}\left[\tanh ^{3}\left(q\left(\zeta-\omega t-\zeta_{0}\right)\right)\right. \\
& \left.-3 \tanh \left(q\left(\zeta-\omega t-\zeta_{0}\right)\right)\right]
\end{aligned}
$$

It can be clearly seen that for this example too our proposed scheme reduces the errors of other methods.

Example 3: The third problem [1] is following Kuramoto-Shivansky equation:

$\frac{\partial \psi}{\partial t}+\psi \frac{\partial \psi}{\partial \zeta}+\frac{\partial^{2} \psi}{\partial \zeta^{2}}+4 \frac{\partial^{3} \psi}{\partial \zeta^{3}}+\frac{\partial^{4} \psi}{\partial \zeta^{4}}=0$

with exact solution

$\psi(\zeta, t)=\omega+9-15\left[\tanh \left(q\left(\zeta-\omega t-\zeta_{0}\right)\right)\right.$

$\left.+\tanh ^{2}\left(q\left(\zeta-\omega t-\zeta_{0}\right)\right)-\tanh ^{3}\left(q\left(\zeta-\omega t-\zeta_{0}\right)\right)\right]$

and initial condition

$$
\begin{aligned}
& \psi(\zeta, 0)=\omega+9-15\left[\tanh \left(q\left(\zeta-\zeta_{0}\right)\right)\right. \\
& \left.+\tanh ^{2}\left(q\left(\zeta-\zeta_{0}\right)\right)-\tanh ^{3}\left(q\left(\zeta-\zeta_{0}\right)\right)\right]
\end{aligned}
$$


The absolute errors after applying different techniques are given in Table-5 at some arbitrary points.

Table-5: Error analysis of Example-3 in first iteration

\begin{tabular}{|l|l|l|l|}
\hline$(\mathrm{x}, \mathrm{t})$ & $(0.1,0.2)$ & $(0.3,0.5)$ & $(0.2,0.6)$ \\
\hline VIM & $1.52223 \times 10^{-6}$ & $\begin{array}{l}4.35347 \times \\
10^{-6}\end{array}$ & $\begin{array}{l}8.48329 \times \\
10^{-6}\end{array}$ \\
\hline MVIM & $2.26747 \times 10^{-7}$ & $\begin{array}{l}2.18233 \times \\
10^{-6}\end{array}$ & $\begin{array}{l}5.3012 \times \\
10^{-6}\end{array}$ \\
\hline MVIM+PADE & $2.26746 \times 10^{-7}$ & $\begin{array}{l}2.18227 \times \\
10^{-6}\end{array}$ & $\begin{array}{l}5.30119 \times \\
10^{-6}\end{array}$ \\
\hline MVIM+RAT & $2.23712 \times 10^{-7}$ & $\begin{array}{l}2.16956 \times \\
10^{-6}\end{array}$ & $\begin{array}{l}5.28483 \times \\
10^{-6}\end{array}$ \\
\hline & & & \\
MVIM+ECORAT & $2.23229 \times 10^{-7}$ & $2.17045 \times$ & $\begin{array}{l}5.28524 \times \\
10^{-6}\end{array}$ \\
& & & \\
\hline
\end{tabular}

It can be seen from the tables that proposed approach is giving better accuracy than the mentioned methods. In [1], original VIM is applied in all three problems solved above and it is obvious from the results that proposed approach can be preferred to solve them.

\section{Conclusion}

In this paper, a coupled approach to solve KuramotoShivashinky equation has been proposed. In this approach, Modified variational iteration method (MVIM) is applied first and then to increase the region of convergence rational approximations by MATHEMATICA are used. Results given in the tables indicate that proposed approach is giving slightly better results than previously used methods as mentioned above. There are many options available for rational approximation in MATHEMATICA which can be opted according to the problems in consideration. This method is easy, fast, efficient and can be used in variety of problems [25-27] arising in science and engineering.

\section{References}

[1]Mehdi Gholami Porshokouhi, Behzad Ghanbari, Application of He's variational iteration method for solution of the family of KuramotoSivashinsky equations, Journal of King Saud University (2011), 23, 407-411.

[2]Tamer A. Abassy, Magdy A. El-Tawil, H.El Zoheiry,Toward a modified variational iteration method, Journal of Computational and Applied Mathematics (2007), 207(1), 137-147.

[3]Tamer A. Abassy, Magdy A. El-Tawil, H.El Zoheiry, Solving nonlinear partial differential equations using the modified variational iteration Padé technique, Journal of Computational and Applied Mathematics (2007), 207(1), 73-91.

[4]Tamer A. Abassy, Magdy A. El-Tawil, H. ElZoheiry, Modified variational iteration method for Boussinesq equation, Computers and Mathematics with Applications (2007), 54, 955-965.

[5]Tamer A. Abassy, Modified variational iteration method (nonlinear homogeneous initial value problem), Computers \& Mathematics with Applications (2010), 59(2), 912-918.

[6]Y. Kuramoto, T. Tsuzuki, Persistent propagation of concentration waves in dissipative media far from thermal equilibrium, Prog. Theor. Phys. (1976), 55(2), 356-369.

[7]Y. Kuramoto, Diffusion induced chaos in reaction systems, Progr. Theoret. Phys. Suppl. (1978), 64, 346-367.

[8]Y. Kuramoto, Chemical Oscillations Waves and Turbulence,(1984), Springer, Berlin.

[9]Kuramto-Sivashinsky equation:http://people.maths.ox.ac.uk/trefethen/pd ectb/kuramoto2.pdf.

[10] G. I. Sivashinsky, Nonlinear analysis of hydrodynamics instability in laminar flames, Part I: Derivations of the basic equations, Acta Astronauica (1977), 4, 1176-1206.

[11] G. I. Sivashinsky, D. M. Michelson, On Irregular Wavy Flow of a Liquid Film Down a Vertical Plane, Prog. Theor. Phys. (1980), 63 (6), 21122114.

[12] G. I. Sivashinsky, Instabilities, pattern formation and turbulence in flames, Ann. Rev. Fluid Mech.(1983), 15, 179-199.

[13] J.H. He, Variational iteration method: a kind of nonlinear analytical technique: some examples. Int. J. Nonlinear Mech.(1999),34(4), 699-708.

[14] J. H. He, Variational iteration method-Some recent results and new interpretations, Journal of Computational and Applied Mathematics (2007), 207(1), 3-17.

[15] J. H. He, X. H. Wu, Variational iteration method: New development and applications, Computers \& Mathematics with Applications (2007), 54(7-8), 881-894. 
[16] J.H. He, G. C. Wu, F. Austin, The Variational Iteration Method Which Should Be Followed, Nonl. Sci. Lett. A (2010), 1(1), 1-30.

[17] Martin Rost, Joachim Krug, A particle model for the Kuramoto-Sivashinsky equation, Physica $D$ (1995), 88, 1-13.

[18] F. Geng, A modified variational iteration method for solving Riccati differential equations, Computers \& Mathematics with Applications (2010), 60(7), 1868-1872.

[19] L. A. Soltani, A. Shirzadi, A new modification of the variational iteration method, Computers \& Mathematics with Applications (2010), 59(8), 2528-2535.

[20] S. T. Mohyud-Din, M. A. Noor, Modified variational iteration method for solving Fisher's equations, Journal of Applied Mathematics and Computing (2009), 31(1-2), 295-308.

[21] M. Pathak, P. Joshi, High order numerical solution of a Volterra integro-differential equation arising in oscillating magnetic fields using variational iteration method, International Journal of Advanced Science and Technology (2014), 69, 47-56.

[22] M. Pathak, P. Joshi, A high order solution of three dimensional time dependent nonlinear convective-diffusive problem using modified variational iteration method, Computers \&
Mathematics with Applications 63 (1), 25-35 (2015), 8(1), 1-5.

[23] C. Yang, J. Hou, Numerical solution of integrodifferential equations of fractional order by Laplace decomposition method, WSEAS Trans. Math., 12, No 12 (2013), 1173-1183.

[24] M. Inokuti, H. Sekine,T. Mura, General use of the Lagrange multiplier in non-linear mathematical physics, in: S. Nemat-Nasser (Ed.),Variational Method in the Mechanics of Solids, Pergamon Press, Oxford(1978),156-162.

[25] G. Mircea, M. Neamt,u, A.L. Ciurdariu, D. Opris, Numerical simulations for dynamic stochastic and hybrid models of internet networks, WSEAS Trans. Math.(2009), 12(8), pp. 679-688.

[26] M Pathak, P Joshi, Numerical Solution of Acoustic Wave Equation Using Method of Lines, World Journal of Modelling and Simulation (2018), 14, 243-256.

[27] P Joshi, M Pathak, A Coupled Approach for Solving a Class of Singular Initial Value Problems of Lane-Emden Type Arising in Astrophysics, Harmony Search and Nature Inspired Optimization Algorithms (2019), 669678

\section{Creative Commons Attribution License 4.0 (Attribution 4.0 International, CC BY 4.0)}

This article is published under the terms of the Creative Commons Attribution License 4.0 https://creativecommons.org/licenses/by/4.0/deed.en_US 